\section{Heterogeneity in Microsatellite Instability in Metastatic Colorectal Cancer: Mechanisms and Clinical Implications}

\author{
Rona Yaeger, MD
}

$\mathrm{T}$ argeted therapy has exposed a high degree of tumor heterogeneity in colorectal cancer (CRC). Compared with other cancer types, patients with CRC exhibit a relatively short duration of response to targeted therapy, and individual patients often harbor a striking number of resistance alterations. ${ }^{1,2}$ Tumor heterogeneity has thus been proposed as the key mechanism underlying incomplete response of CRCs to therapy. Analysis of paired primary tumors and metastases shows a high degree of concordance of genomic alterations, particularly for known oncogenic drivers. $^{3-5}$ This suggests that the genomic alterations required for migration and metastasis of CRC occur early and can be found in the primary tumor. 6 Therefore, despite the existence of clonal heterogeneity, there is a dominant single clone and, for clinically significant genomic drivers, analysis of the primary or the metastatic cancer is appropriate. In a study published in this issue of JNCCN, He et al ${ }^{7}$ examined for heterogeneity in microsatellite instability (MSI) status between primary and metastatic sites and among different metastatic sites. This question is clinically important and timely because immune checkpoint inhibitors, such as pembrolizumab and nivolumab, have had dramatic efficacy in this group of cancers and have changed the treatment paradigm for these tumors. ${ }^{8-10}$

He et $\mathrm{al}^{7}$ examined paired primary and metastatic CRC from 369 patients and evaluated MSI status using immunohistochemistry. They confirmed MSI-high (MSI-H) cases using polymerase chain reaction. They found that in 46 patients with an MSI-H primary tumor, 37 (80\%) had an MSI-H metastasis and 9 (20\%) had a microsatellite stable (MSS) metastasis. All of the discrepant cases involved peritoneal or ovarian metastatic sites; the authors found complete concordance in liver, lung, and distant lymph nodes. As could be expected, in the cases with discordance, the MSS specimen exhibited a lower tumor mutational burden than the corresponding MSI-H primary tumor. These differences were also associated with corresponding biologic changes; the MSS metastases exhibited significantly less lymphocyte infiltration compared with the corresponding MSI-H primary tumors. Among the 321 patients with MSS tumors, more than $98 \%$ of patients with an MSS primary tumor had MSS metastasis. For patients with MSI-H metastases, approximately $90 \%$ had MSI-H primary sites.

Further analysis of the MSI-H cases was performed to determine the etiology of MSI. Of the 46 patients with MSI-H primary sites, 13 had germline mutations detected in mismatch repair genes, and these patients showed $100 \%$ concordance between the primary and metastatic tumors for MSI status. Two patients had concurrent BRAF V600E mutation, suggesting sporadic MSI from silencing MLH1 gene expression. These patients also both showed concordance for MSI status between the primary and metastatic tumors. The remaining 31 patients with mutation-negative disease showed no identifiable germline alteration or BRAF V600E mutation. These included all discordant cases.

How can we mechanistically interpret what is happening with MSI status in CRC? The heterogeneity in MSI status appears to be due to timing of the development of

See page 1174 for related article.

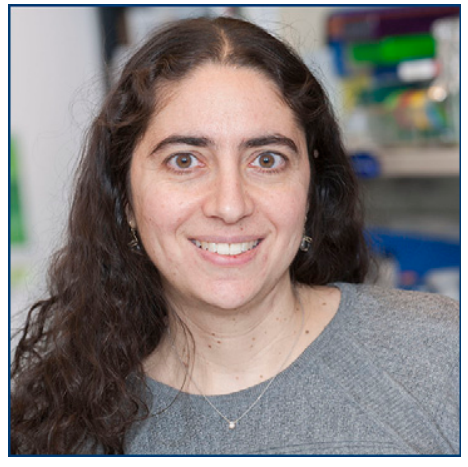

RONA YAEGER, MD

Rona Yaeger, MD, is Associate Director of the Colorectal Cancer Research Center and an Assistant Attending Physician in the Department of Medicine at Memorial Sloan Kettering Cancer Center. Her clinical and laboratory research center on understanding molecular subsets of colorectal cancer and anal squamous cell cancer and developing new therapies based on this understanding.

doi: $10.6004 /$ jnccn.2019.7348

The ideas and viewpoints expressed in this commentary are those of the author and do not necessarily represent any policy, position, or program of NCCN. 
MSI in the tumor. MSI appears to occur early with a higher rate of concordance of primary tumor for metastases that are MSI-H. In contrast, metastases of MSI-H primary sites show a higher discordance. In these cases, some metastases seem to have diverged before the development of MSI. These discordant metastases probably exhibit a lower tumor mutation burden because they had not yet acquired all the mutations seen in the primary before metastatic seeding.

These findings are consistent with a recent study suggesting that metastatic seeding in CRC may occur before clinical detectability. ${ }^{4}$ Interestingly, none of these cases are due to germline alterations or MLH1 hypermethylation associated with BRAF V600E mutation. MSI in these cases may result from false-positivity of the primary site for $\mathrm{MSI}-\mathrm{H}$, the presence of occult germline mutation, or biallelic somatic mutations in a mismatch repair gene. For double somatic mismatch repair gene inactivation, the probability of developing MSI-H increases over time as the chance of hitting a mismatch repair gene increases. This potentially explains how the MSI alteration occurs later and after some clones may have already gained the ability to metastasize.

Although the numbers in this study are small, ${ }^{7}$ they show an intriguing suggestion of differences in MSI status of metastases by site. Metastases that could develop by direct shedding into the abdomen-peritoneal and ovarian metastases-are most commonly discordant. In contrast, metastatic sites that require the metastatic clone to travel through the blood or lymphatic system, through a new environment, to invade distant sites-liver, lungs, and distant lymph nodes—were all concordant. Therefore, "easy to metastasize sites" may possibly be colonized by early clones, including some whose primary clonal population continues to evolve and gain MSI-H by somatic alterations, and "difficult to metastasize sites" possibly involve later clones that have already acquired MSI-H. A patient in this study ${ }^{7}$ with an MSI-H primary tumor had an MSI-H liver metastasis and MSS peritoneal metastasis, providing support for different mechanistic and temporal pathways for metastases in these different sites.
This study suggests 2 key clinical implications for care of patients and application of immune checkpoint inhibitors. ${ }^{7}$ First, patients should be eligible for immune checkpoint blockade based on MSI testing of either the primary tumor or a metastasis; testing both sites is not necessary. This study confirms that an MSS primary is highly unlikely to be associated with an MSI-H metastasis ( $<2 \%$ chance). This high accuracy of MSI testing establishes that there is no reason to test a metastatic sample for MSI if the primary tumor is MSS. Conversely, this study clarifies that if the metastatic site is MSS, testing the primary tumor would not be helpful because an MSS metastasis, even if associated with an MSI-H primary tumor, exhibits biological features-low tumor mutation burden and decreased lymphocyte infiltration-that suggest immune checkpoint inhibition is unlikely to be effective. Because metastases are the primary cause of mortality, immune checkpoint blockade must have activity against metastases to be clinically meaningful. Second, at this time, we should continue to offer immune checkpoint inhibitors to patients with MSI-H metastatic CRC, irrespective of the spectrum of metastatic involvement. However, this study provides a mechanistic explanation for discordant responses that can occur at different sites with immune checkpoint inhibitors.

Although He et al's work provides important insight into the development of MSI in CRC, some key questions remain. More in-depth analysis of paired MSI-H metastatic CRC samples, possibly with next generation sequencing, is needed to confirm that discordance is due to different subclones rather than to different tumors altogether. Further, in recognition of the limited sample size of this study, investigations in larger datasets are needed to further evaluate patterns of discordance in MSI status by metastatic site and how this affects response to immunotherapy. Finally, further analysis of the interesting subset of MSI-H CRCs resulting from biallelic somatic mutations are needed to verify if these tumors exhibit a larger degree of heterogeneity for MSI and the implications of this heterogeneity on effective application of immunotherapies.

\section{References}

1. Corcoran RB, Andre T, Atreya CE, et al. Combined BRAF, EGFR, and MEK inhibition in patients with BRAF(V600E)-mutant colorectal cancer. Cancer Discov 2018;8:428-443.

2. Siravegna G, Lazzari L, Crisafulli G, et al. Radiologic and genomic evolution of individual metastases during HER2 blockade in colorectal cancer. Cancer Cell 2018;34:148-162.

3. Brannon AR, Vakiani E, Sylvester BE, et al. Comparative sequencing analysis reveals high genomic concordance between matched primary and metastatic colorectal cancer lesions. Genome Biol 2014;15:454

4. $\mathrm{Hu} Z$, Ding J, Ma Z, et al. Quantitative evidence for early metastatic seeding in colorectal cancer. Nat Genet 2019;51:1113-1122.

5. Yaeger R, Chatila WK, Lipsyc MD, et al. Clinical sequencing defines the genomic landscape of metastatic colorectal cancer. Cancer Cell 2018;33: 125-136.

6. Ryser MD, Min BH, Siegmund KD, et al. Spatial mutation patterns as markers of early colorectal tumor cell mobility. Proc Natl Acad Sci U S A 2018;115:5774-5779.

7. He WZ, Hu WM, Wang F, et al. Comparison of mismatch repair status between primary and matched metastatic sites in patients with colorectal cancer. J Natl Compr Canc Netw 2019;17:1174-1183.

8. Le DT, Durham JN, Smith KN, et al. Mismatch repair deficiency predicts response of solid tumors to PD-1 blockade. Science 2017;357:409-413.

9. Overman MJ, McDermott R, Leach JL, et al. Nivolumab in patients with metastatic DNA mismatch repair-deficient or microsatellite instabilityhigh colorectal cancer (CheckMate 142): an open-label, multicentre, phase 2 study. Lancet Oncol 2017;18:1182-1191.

10. Overman MJ, Lonardi S, Wong KYM, et al. Durable clinical benefit with nivolumab plus ipilimumab in DNA mismatch repair-deficient/microsatellite instability-high metastatic colorectal cancer. J Clin Oncol 2018;36:773-779. 